\title{
A Cooperative Vehicular Network Framework
}

\author{
Oscar Trullols-Cruces, Julian Morillo-Pozo, Jose M. Barcelo, Jorge Garcia-Vidal \\ Department of Computer Architecture (DAC), UPC, Barcelona, Spain \\ $\{$ trullols,jmorillo,joseb,jorge\}@ac.upc.edu
}

\begin{abstract}
Vehicular Ad Hoc Networks are networks characterized by intermittent connectivity and rapid changes in their topology. This paper addresses car-to-road communications in which vehicles use Access Points (AP) in a Delay Tolerant Network architecture. Results show how the combination of a Delay-Cooperative ARQ mechanism reduces packet losses and in conjunction with a Carry-and-Forward cooperative mechanism improves performance parameters in terms of total file transfer delay and number of AP needed to download files.
\end{abstract}

Index Terms-Cooperative networks, VANET, Delay Tolerant Networks.

\section{INTRODUCTION}

Vehicular Ad-hoc NETworks (VANET) are a particular case of MANET in which nodes are vehicles that move following specific patterns (i.e. roads). VANETs are networks characterized by intermittent connectivity and rapid changes in their topology. In contrast with other ad-hoc networks, these networks also have very specific mobility patterns. As an example consider vehicles traveling on highways that have sparse Access Points (AP) distributed along the route. Due to the high speeds, vehicles have few seconds to access Internet or other vehicles to which may want to communicate. Furthermore, the environment presents a high level of packet losses. For example, measurements of UDP and TCP transmissions of vehicles in a highway passing in front of an AP moving at different speeds, report losses on the order of 50$60 \%$ depending on the nominal sending rate and vehicle speed, [1]. In ranges of around 250 meters, throughput reaches approximately $4 \mathrm{Mb} / \mathrm{s}$, while at larger distances (e.g. 400 meters) the throughput drops to around $1 \mathrm{Mb} / \mathrm{s}$.

We consider vehicles that may download information from an infrastructure network. Vehicles access this network through a sequence of APs located along roads (highway scenarios) or streets (urban scenarios). These APs do not cover the whole path followed by the vehicle, facing this one a scenario with sparse opportunities to download information. In this paper, we define a framework that handles efficiently the downloading of files from infrastructure located along the road, following a Delay Tolerant Network (DTN) architecture with APs acting as custodians. Each time a vehicle enters an AP coverage area, it registers its location and downloads blocks of packets from the AP. From vehicle location registration, a scheduler plans the next AP that the vehicle visits. The aim of the framework is to exploit the time while cars are in the dark areas between APs in order to improve throughput and file transfer delay. To accomplish this, the framework incorporates two mechanisms: Delayed Cooperative ARQ (DC-ARQ) to recover packet losses and a Carry and Forward (C\&F) mechanism from vehicles traveling in opposite direction.
The main contributions of this paper are:

- Definition of a Vehicular Ad Hoc Network framework following a DTN architecture that opportunistically allows downloading packets when vehicles cross AP.

- Integration in the VANET framework of (i) a Delayed Cooperative ARQ (DC-ARQ) mechanism to reduce packet losses of transmissions from AP to vehicles and from vehicles to vehicles and (ii) a carry-and-forward mechanism based on the predictability of vehicle road routes that cross opportunistically other vehicles to improve transfer delivery of information.

- To evaluate the proposal, we have completed an experimental work reported in [4] (in which we built a prototype in three cars to check the feasibility of the DC-ARQ mechanism in an urban scenario) with simulations to study the proposal in a more scalable and general scenario.

The paper is organized as follows: Firstly, we discuss the related work. Secondly, we describe the proposed integrated vehicular framework. Thirdly, a description of the experimental scenario and settings is outlined. Fourthly, we discuss the obtained results. Lastly, we draw some conclusions, open issues and further work.

\section{RELATED WORK}

A performance study in terms of losses when vehicles enter the coverage of an access point in a highway and exchange UDP and TCP packets is presented in [1], reporting losses on the order of $50-60 \%$ depending on the nominal sending rate and vehicle speed.

Cooperative ARQs are schemes which increase link reliability in data link protocols through the use of node cooperation; [2], [3], [5], [6] and [7]. The work reported in [2] presents a novel frame exchange mechanism between a node and its cooperators called C-ARQ/FC (Cooperative ARQ with Frame Combining). This exchange method exploits the strong correlation between erroneous frames received by different nodes in order to compress the information exchange, thus reducing considerably the coordination overhead.

Most of the work related to opportunistic vehicular networks deal with opportunistic forwarding strategies, in which nodes schedule the forwarding of packets according to opportunities; [8], [9] and [10]. This scheduling may be based on: historical path likelihoods, [8], packet replication, [9], or on the expected packet forwarding delay, [10]. These proposals take as a point of reference epidemic routing [11]. Their main

This work has been supported by Spanish Ministry of Science and Technology under grant TSI2007-66869-C02-01 and EuroNF Network of Excellence. 
objective is to optimize contact opportunities to forward packets in intermittent scenarios, but they do not consider how to optimize the transference of such information given that you have contacted another node. SPAWN, [12], is a cooperative strategy for VANETs. However, SPAWN proposes a gossip mechanism that propagates content information while our proposal main objective is improving file transfer delay and number of APs to download a specific file. Finally, Delay Cooperative-ARQ (DC-ARQ), [4], is proposed in a Vehicular Network where experimentally the benefits in terms of packet loss ratio reduction are shown. The experiments were performed in an urban scenario using three vehicles (two of them cooperators) and one AP. UDP packets were sent from the AP to each vehicle, and was shown how a simple DC-ARQ scheme was able to minimize efficiently packet losses

\section{VEHICULAR FRAMEWORK}

We consider vehicles that download information from infrastructure located along roads, see Fig. 1. This infrastructure does not cover the whole path followed by the vehicle, thus having an intermittent connectivity. Access Points (AP) are connected via a backbone. Each time a vehicle reaches coverage of an $\mathrm{AP}$ (i.e. $\mathrm{AP}_{\mathrm{k}}$ ), registers its direction, velocity and identity in a server. We assume that the first time a vehicle enters the network, obtains identification (Node-ID). It is not the purpose of this paper to define a naming or identification mechanism, neither to define the backbone network.

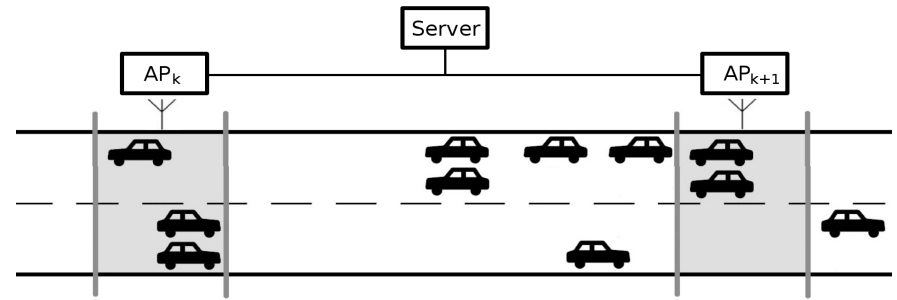

Figure 1. Network scenario.

Due to the intermittent connectivity and the harsh physical conditions, TCP/IP provides low data transfer to vehicles. We propose a Delay Tolerant Vehicular framework to handle intermittent connectivity where Access Points, acting as custodians, form a Delay Tolerant Network (DTN), [13]. This means that access points have storing capabilities and are able to store a whole file or blocks of files. The server schedules which AP stores files or blocks of files that have to be transferred to vehicles (Fig. 1). Simple scheduling decisions can be taken from the registered information delivered by vehicles each time they enter AP coverage. The server transfers packets to AP custodians using TCP/IP stack. In highway scenarios in which vehicles follow the same direction for long periods, the server predicts which will be the next AP on the highway to be visited by the vehicle and then transfers the file or unacknowledged blocks of the file to that AP.

Access Points (custodians in DTN terminology) divide files in blocks of size $\mathrm{L}_{\mathrm{B}}$, having thus $B=\left\lceil N / L_{B}\right\rceil$ blocks per file, where $\mathrm{N}$ is the size in bytes of the file. Each block will contain P packets of size $L_{p}$, the L2 MTU (Maximum Transfer Unit).
Vehicles that want to download a file send a query to the server. From the vehicle registered information, the server predicts to which AP has to send the file. We may conclude two vehicle location situations: (i) a vehicle is in $\mathrm{AP}_{\mathrm{k}}$ coverage, or (ii) a vehicle has left $\mathrm{AP}_{\mathrm{k}}$ coverage area and is driving from the last registered direction at average velocity $v_{k}(\mathrm{~km} / \mathrm{h})$. Without any optimized scheduling discipline defined in the server, next time the vehicle enters $\mathrm{AP}_{k+1}$ coverage, it registers, updates its location and velocity information and continues downloading packets. The vehicle lasts an average of $T_{k}=d_{k} / v_{k}$ seconds to cross $\mathrm{AP}_{\mathrm{k}}$ coverage area (where $\mathrm{d}_{\mathrm{k}}$ is the AP coverage in $\mathrm{km}$ ) and is able to download $N_{k}=T_{k} \cdot V t_{k}$ bytes assuming an average throughput of $V t_{k}$ bytes/s when traversing $\mathrm{AP}_{\mathrm{k}}$. If the file size is $\mathrm{N}$ bytes, the vehicle will need " $K$ " access points where " $K$ " is that $k$ index that makes $N_{l}+\ldots+N_{K}=N$. Furthermore the average time $T$ needed to download the file is:

$$
T \leq \sum_{k=1}^{K} T_{k}+\sum_{k=1}^{K-1} \frac{D_{k}-\left(d_{k}+d_{k+1}\right) / 2}{v_{k}}+\frac{D_{0}}{v_{0}}
$$

where we have considered without any loss of generality that the vehicle travels at average velocity $v_{k}$ from $\mathrm{AP}_{\mathrm{k}}$ to $\mathrm{AP}_{k+1}$ and the distance from $\mathrm{AP}_{k}$ to $\mathrm{AP}_{k+1}$ is $D_{k} \mathrm{~km} . \mathrm{D}_{0} / \mathrm{v}_{0}$ is the time lasted from the initial query was issued to the registration in the first AP and the reason of the inequality is due to the fact that in the last AP, the vehicle may finish downloading the file before leaving AP coverage. We should note that term $D_{k} / v_{k}$ dominates transfer delay (i.e. time spent traveling from one access point to the next one) and throughput (opportunity to download) is a scarce resource. Only, when the vehicle speed is low and the number of downloading nodes also is low, transfer delay will be dominated by $T_{k}$ (i.e. $T \leq D_{0} / v_{0}+T_{0}$ ) and the vehicle downloads the file with only one AP. Therefore, any technique aimed to (i) maximize transfer opportunities when vehicles cross AP coverage and (ii) use of gap areas (i.e. time spent traveling between AP) will improve global transfer delays.

To fulfill these objectives, our framework reduces packet losses and optimizes global transfer delay using the following mechanisms: (i) an end-to-end ARQ scheme with custodians to provide end-to-end reliability; (ii) a Delay Cooperative ARQ (DC-ARQ) scheme with neighbor vehicles after leaving AP coverage (gap areas) to minimize packet losses; and (iii) a carry and forward scheme in vehicles traveling in opposite direction that opportunistically will cross with the vehicle in the road to improve total transfer delay.

\section{A. End-to-end $A R Q$}

The framework needs an end-to-end ARQ mechanism that operates at transfer opportunities. An end-to-end ARQ with several coordination packets would waste resources. As stated by K. Fall in [13], the use of TCP or a TCP based modification would severely limit the throughput; the network would last too long to start a transmission, or would use bandwidth in retransmissions for nodes that are already out of coverage. To combat these harmful effects we have applied DTN-based solutions.

Our solution consists of vehicles broadcasting periodically beacons registering location and velocity. Custodians use this beacon to detect the vehicle presence and as indication of 
transfer opportunity. Beacons are also used as end-to-end-ARQ with custodians indicating which packets are lost and thus should be retransmitted. Beacons only indicate lost packets belonging to some non-completely received blocks. Custodians do not retransmit lost packets immediately, but take notice of them, and continue transmitting the packets of the file sequentially. After finishing the first round of the file, a round of retransmissions starts, and new rounds of retransmissions will be done until the destination completes the bundle or it runs out of coverage. Using this mechanism the expected behavior of the framework is shown at Fig. 2(a). The download starts in one AP and could be continued at the followings AP.

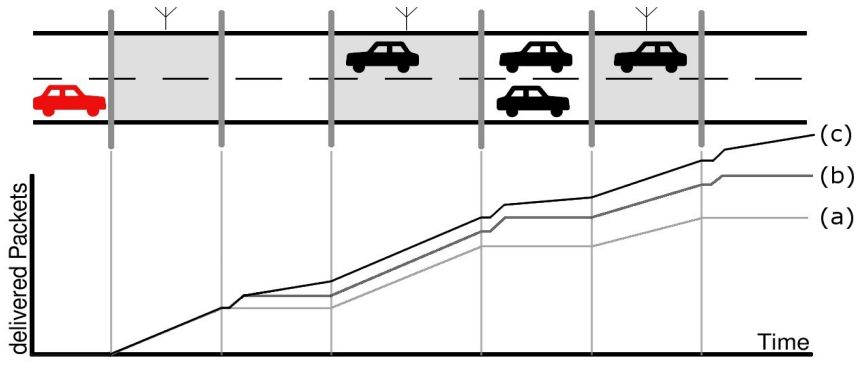

Figure 2. Figure 1 Expected framework behavior using: (a) End-to-End ARQ, (b) adding DC-ARQ, (c) adding C\&F

\section{B. Delayed Cooperative ARQ (DC-ARQ)}

Due to the harsh conditions in which nodes communicate, packet losses are on the order of $50-60 \%,[1]$ and [4]. In paper [4], we showed that packet losses could drastically be reduced using a Delayed Cooperative ARQ (DC-ARQ) mechanism, improving the throughput obtained by vehicles when downloading information from an AP. DC-ARQ works as follows (see [4] for a more detailed description of the general mechanism operation): vehicles traveling in the same platoon can be elected as DC-ARQ cooperators. Election is performed based on a Hello protocol. Hello headers indicate the nodes willingness to participate as cooperators. DC-ARQ cooperation means that cooperators will buffer packets destined to other vehicles of its own platoon. When vehicles leave the AP coverage area, they enter into a Cooperative-ARQ phase in which they recover the lost packets that have been received by its DC-ARQ cooperators. Note that in contrast with C-ARQ in [2], cooperation is not immediately performed packet by packet at layer 2. DC-ARQ is performed above layer 3. The reason is that the framework objective is to optimize contact opportunities minimizing the number of retransmitted packets with custodians. After leaving the AP, vehicles have no opportunities to go on downloading packets until they meet next custodians. Therefore, vehicles use this traveling time to cooperate between themselves in order to reduce the amount of packets that will have to be retransmitted when they enter the next coverage custodian. It is important to note that this mechanism also works if vehicles traveling in opposite direction exchange packets among themselves.

Adding this mechanism to the framework the expected behavior is shown at Fig. 2(b). When a node has left AP coverage cooperates with the platoon and recovers some packets.

\section{Carry-and-forward $(C \& F)$ in opposite directions}

The server knows, from the registered information, which AP will be visited by each vehicle. In a highway/road scenario, vehicles follow the road for long distances. In case there is a cross-point, the number of possible next AP that the vehicle will encounter is reduced. The server uploads the file to future possible APs. These APs will be the next information custodians. In case a vehicle does not cross the AP in a reasonable amount of time, the AP removes the file. APs may also delete files directed to vehicles that have already left AP coverage. More intelligent predictions may be obtained if vehicles pre-register their traveling destination (current GPS devices calculate optimal traveling routes and organize vehicle path-road).

Vehicles passing through APs may further cooperate carrying and forwarding packets directed to vehicles that they would encounter in the road. The concept is related to epidemic routing, [11], and PRoPHET protocol, [14]. In highway/road scenarios, vehicles traveling in opposite direction will encounter each other with high probability. A scheduler defines a delivery discipline to these Carry-and-Forward-Cooperators in which blocks of packets are selected to be carried-andforwarded to other vehicles.

Let $S_{k}(t)$ be the number of vehicles crossing $A P_{k}$ coverage area at time $t$. $S_{k}$ is obtained from periodic beacons sent by vehicles and can be expressed as $S_{k}(t)=S_{k} \rightarrow(t)+S_{k}^{\leftarrow}(t)$, where the arrows indicate the vehicles traveling directions. Furthermore, $A P_{k}$ receives notification from neighbor AP which vehicles will arrive to its coverage. Let us call $\Omega_{\mathrm{k}}$ the set of $\mathrm{AP}_{\mathrm{k}}$ neighbors and $M_{\omega}(t)$, being $\omega \in \Omega_{\mathrm{k}}$, the number of vehicles traveling from $A P_{\omega}$ towards $A P_{k}$ at time $t$. Therefore, $A P_{k}$ is aware of $\mathrm{K}(\mathrm{t})$ vehicles: those under its coverage area plus those traveling towards its coverage area.

$$
\mathrm{K}(\mathrm{t})=\mathrm{S}_{\mathrm{k}}(\mathrm{t})+\mathrm{S}_{\mathrm{k}}^{\leftarrow}(\mathrm{t})+\sum_{\omega \in \Omega} \mathrm{M}_{\omega}(\mathrm{t})
$$

Clearly, $\mathrm{S}_{\mathrm{k}} \rightarrow(\mathrm{t})$ (respectively $\left.\mathrm{S}_{\mathrm{k}} \leftarrow(\mathrm{t})\right)$ are candidates to carryand-forward packets to those vehicles traveling towards $\mathrm{AP}_{\mathrm{k}}$. The framework uses the $\mathrm{S}_{\mathrm{k}} \rightarrow(\mathrm{t})$ (respectively $\mathrm{S}_{\mathrm{k}} \leftarrow(\mathrm{t})$ ) to carry and forward packets to $\mathrm{M}_{\omega}(\mathrm{t})$ when there are no vehicles downloading packets at $\mathrm{AP}_{\mathrm{k}}$.

A node cooperates when it is possible to do it without interfering with nodes that are currently downloading in the same AP. That means that when there are no vehicles downloading packets in $A P_{k}$, this one schedules packets to be carried and forwarded towards some of the $M_{\omega}(t)$ vehicles, being $\omega \in \Omega_{k}$.

We propose two versions of this mechanism. In the first one, each time that cooperation is possible, the AP chooses the destination among the $\mathrm{M}_{\omega}$ vehicles, the block that will be cooperated, and a cooperator among the $S_{k}$ vehicles. In the second version, each time that cooperation is possible, the AP chooses the destination and the block but broadcasts the packets in order to allow all the nodes on coverage to cooperate with the destination. There is not a big difference between the packets received by the cooperator in the first version and the packets received between all of them in the second, because 
DC-ARQ would also recover C\&F cooperation packets before crossing the destination. The main difference is that in the second version the cooperation is widespread between several vehicles and the destination node will have more opportunities to get the packets. After adding this mechanism to the framework, the expected behavior is shown at Fig. 2(c). When a node is traveling between APs will receive C\&F packets improving its total throughput.

In this way, end-to-end-ARQ recovers lost packets without immediate packet retransmissions if there are other packets to transmit, giving a chance to DC-ARQ to recover packets from vehicles that travel in the same platoon when they are out of coverage and the bandwidth is not a scarce resource. And C\&F cooperators seize unused AP. So, both cooperating mechanisms are for free, using resources otherwise no one would use, improving AP transfer opportunities.

\section{EXPERIMENTAL SCENARIO}

The simulations were performed with the standard version of the ns-2.31 simulator [15]. Each AP generates packets to vehicles in its coverage area in round robin basis. Vehicles request files of $10 \mathrm{MB}$ and packets have a size of $1 \mathrm{~KB}$.

For simplicity, we consider a lineal road and leave for further study the general case in which the road has bifurcations. Vehicles travel on a two-way highway with two lanes per direction. The road network infrastructure consists of Access Points placed every $6 \mathrm{Km}$. Vehicles move with constant speed, randomly chosen from a uniform distribution between $70-90 \mathrm{~km} / \mathrm{h}$ on the right lane and between $90-120 \mathrm{~km} / \mathrm{h}$ on the left lane. Vehicle density is modeled with exponential distribution of parameter $\lambda_{1}$ vehicles/s in the right lane and $\lambda_{2}$ vehicles/s in the left lane. These rates consider the maximum number of vehicles following vial rules (e.g. security distances of 80 meters traveling at $90 \mathrm{Km} / \mathrm{h}, 100$ meters traveling at 100 $\mathrm{Km} / \mathrm{h}$, etc). Using these consideration, $\lambda_{1}=0.25$ vehicles $/ \mathrm{s}$ in the right lane and $\lambda_{2}=0.2$ vehicles $/ \mathrm{s}$ in the left lane would fill the highway with the maximum number of vehicles traveling at a maximum of $100 \mathrm{Km} / \mathrm{h}$ and at $120 \mathrm{Km} / \mathrm{h}$ at each lane. Higher densities may be achieved lowering vehicle speeds (then the security distance between vehicles should be lower). In the graphs we normalize $\lambda_{\mathrm{i}}(\mathrm{i}=1,2)$ and for clarity we define parameter $\alpha$ as vehicle density, being $\alpha=1$ the higher density corresponding to vehicles with $120 \mathrm{Km} / \mathrm{h}$ as maximum speed at line 1 and $90 \mathrm{Km} / \mathrm{h}$ as maximum speed at line 2 . Lower values of $\alpha$ indicate a decrease in vehicle density.

TABLE I. CONFIGURATION PARAMETERS
\begin{tabular}{|l|l|}
\hline Antenna & Omnidirectional \\
\hline Frequency & $5.9 \mathrm{GHz}$ \\
\hline RxTh & $-95 \mathrm{dBm}$ \\
\hline CSTh & $-96 \mathrm{dBm}$ \\
\hline Antenna Gain & $1 \mathrm{~dB}$ \\
\hline TxPower & $9.95 \mathrm{Bm}$ \\
\hline
\end{tabular}

For the physical layer we consider IEEE802.11a at a rate of $3 \mathrm{Mb} / \mathrm{s}$. Nodes use omnidirectional antennas, see Table I. We have used Nakagami as propagation model. This model is used to predict signal attenuation in fading environments and has already been used in vehicular scenarios, [16].

\section{RESULTS}

In this section, we evaluate the performance of the proposed scheme through the analysis of the results obtained in the simulations. The parameters evaluated are:

- Total delay experienced by a car to download a file.

- Number of APs needed to download a certain file.

- Number of packets received thanks to the use of DCARQ cooperation scheme.

- Number of packets received thanks to the use of the carry-and-forward cooperation mechanism.

We evaluate all these parameters depending on:

- Density of cars in the road, see Table II.

- Density of cars downloading information: $\delta$

TABLE II. SCENARIOS

\begin{tabular}{|l||l|}
\hline Name & $\boldsymbol{\alpha}$ ( Normalized car density) \\
\hline \hline Scen.-A & $1 / 2$ \\
\hline Scen.-B & $1 / 4$ \\
\hline Scen.-C & $1 / 8$ \\
\hline Scen.-D & $1 / 16$ \\
\hline
\end{tabular}

To analyze the enhancements provided by each of these mechanisms, we show different combinations of them, see Table III.

TABLE III. NAMES USED IN PLOTS

\begin{tabular}{|l||c|c|c|c|}
\hline Mechanism & $\begin{array}{c}\text { E2E } \\
\text { ARQ }\end{array}$ & $\begin{array}{c}\text { Delayed } \\
\text { C-ARQ }\end{array}$ & $\begin{array}{c}\text { C\&F } \\
\text { 1Coop }\end{array}$ & $\begin{array}{c}\text { C\&F } \\
\text { multiCoop }\end{array}$ \\
\hline NoCoop & $\mathrm{X}$ & & & \\
\hline DC-ARQ & $\mathrm{X}$ & $\mathrm{X}$ & & \\
\hline C\&F-1Coop & $\mathrm{X}$ & $\mathrm{X}$ & $\mathrm{X}$ & \\
\hline C\&F-multiCoop & $\mathrm{X}$ & $\mathrm{X}$ & & $\mathrm{X}$ \\
\hline
\end{tabular}

Fig. 3 shows delay and number of used APs, when using only the end-to-end ARQ mechanism. We can see how the number of APs (lines) and delay (points) behave similarly. The reason is that the delay to download a file is dominated by the traveling time between consecutive AP as it was explained in section III, even when using C\&F cooperation, it is very difficult to complete the download between two APs; there are usually some packets that could not have been cooperated and the node needs to wait until the next AP to get them. The simulation results behave according to equation (1) where the number of APs needed to download a file was called $K$. All scenarios need higher number of APs to download a file given an increasing percentage $\delta$ of vehicles downloading information. The highest vehicle density (scenario A), the more number of AP are needed and thus the higher the total transfer delay given by equation (1). In the next evaluations we only comment the average number of APs, because it is more general than total delay and could be extrapolated to different scenarios with different distances between APs. 


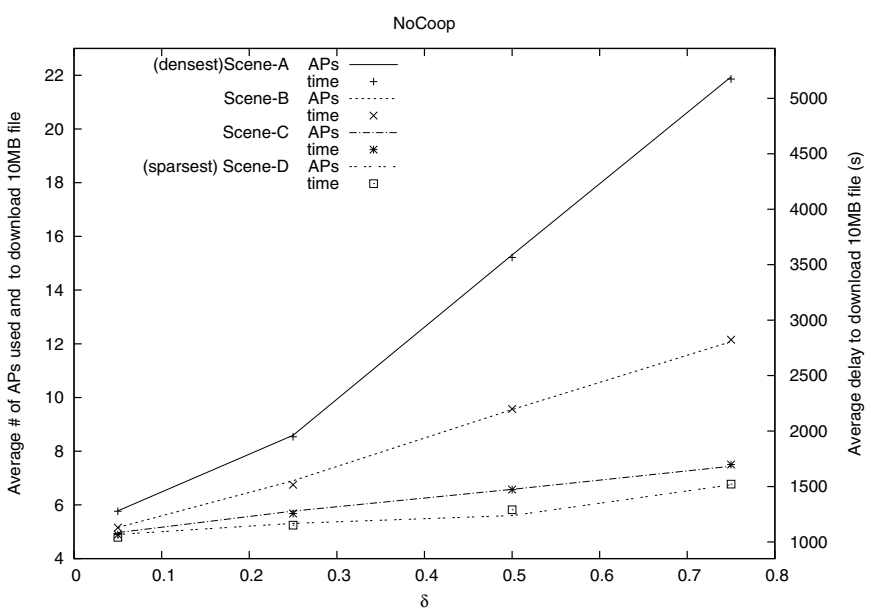

Figure 3. Average \# of used APs and Total Transfer Delay using only EndTo-End ARQ, and no cooperation.

Fig. 4 shows the number of DC-ARQ cooperated packets when using the DC-ARQ mechanism. As expected, there are more DC-ARQ cooperating packets when the car density (scen.-A) is higher, because there are more neighbors in the platoon and the probability that one of them has received the lost packets is higher. However, even in low car density scenarios (scen.-D) where there are few cars to cooperate, DCARQ improves packet loss ratios. This figure is also interesting to see that all the lines are sort of flat, meaning the effectiveness does not vary much when more vehicles start to download. The reason of this behavior is that packet losses and packet recovery does not depend on the number of vehicles downloading information but on vehicle density. This would not be true in case in which vehicles upload information, since collisions would increase packet losses.

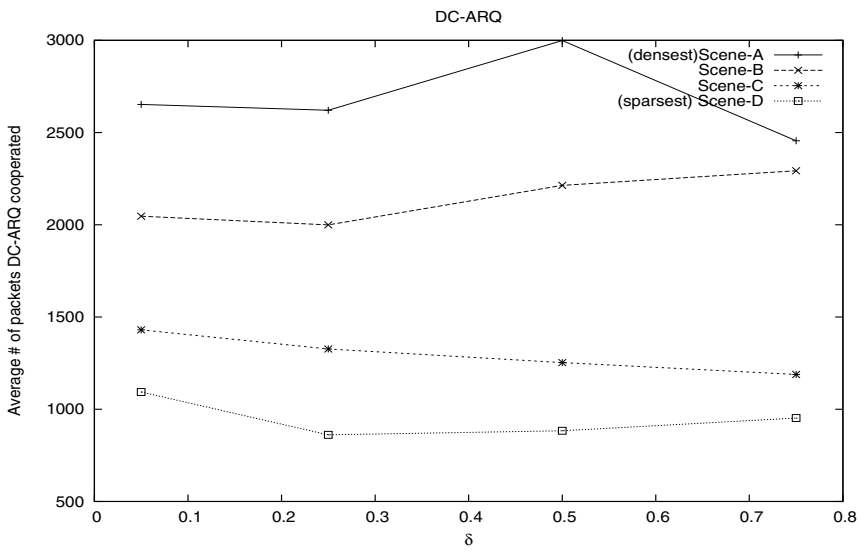

Figure 4. Average \# of DC-ARQ Cooperated packets using DC-ARQ

Fig. 5 shows the mechanism in which a specific vehicle is selected to carry and forward packets (C\&F-1Coop) and Fig. 6 shows the mechanism in which any vehicle traversing the AP may carry and forward packets (C\&F-MultiCoop). At first, It may seem unexpected that for low values of $\delta$, the cooperation increases when $\delta \psi$ increases (note the peaks in figures 5 and 6 ). But when $\delta$ increases and $\delta$ is low $(\delta \leq 0.25)$ the number of APs used to finish a download also increases, some of them will be inactive and therefore there are more cooperation opportunities. However, when the number of nodes downloading files reaches certain point (high $\delta$ values, i.e. $\delta \geq 0.25$ ) is most probably that there are vehicles downloading packets in the AP. Since the strategy is to use AP for cooperation when such AP is not active, high values of $\delta$ imply that there is always activity in the AP. Thus, C\&F cooperation decreases with increasing $\delta$. Paying attention to the different scenarios, in denser scenarios (i.e. scen.-A) we observe that with low $\delta$ have more cooperation opportunities because there may be more possible cooperators than in sparse scenarios (i.e. scen.-D) where there are no vehicles traversing the AP. When the $\delta \psi$ increases, the bandwidth of an AP may be shared among more nodes and $\mathrm{C} \& \mathrm{~F}$ cooperation is not possible without decreasing downloading car throughputs.

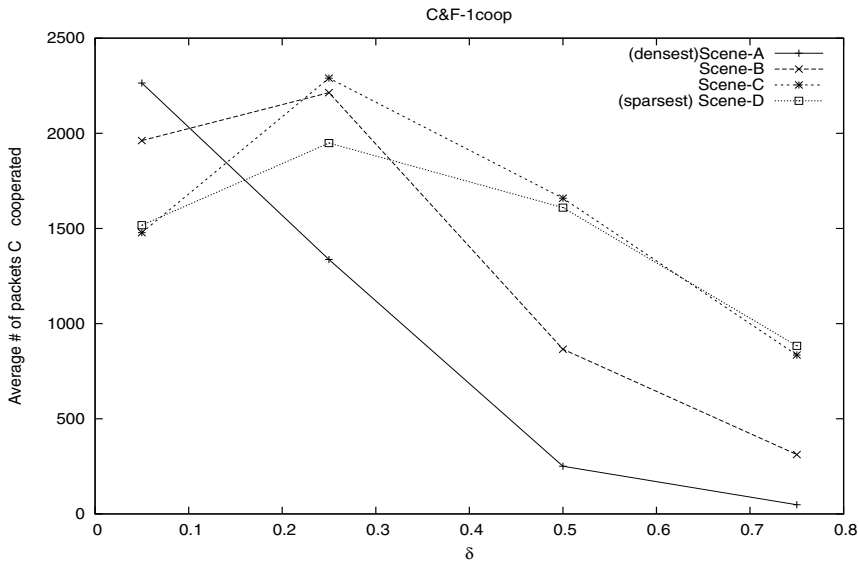

Figure 5. Average \# of C\&F Cooperated packets using C\&F-1Coop

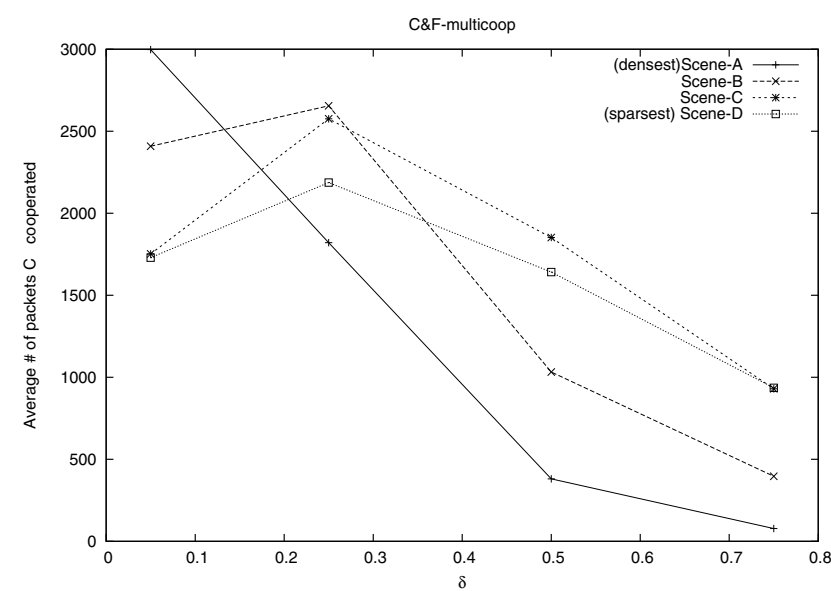

Figure 6. Average \# of C\&F Cooperated using C\&F-MultiCoop

Logically, the multi-cooperative mechanism behaves slightly better since the vehicle traveling in opposite direction will have more opportunities to download packets. In other words, the multi-cooperative mechanism is most robust since more vehicles may carry the same packets.

Fig. 7 and Fig. 8 shows the average number of used APs for the densest scenario (scen.-A) and the sparsest scenario (scen.D) using the combination of the different mechanism, see 
Table III. DC-ARQ cooperation has a high impact in the performance, which is better when using $\mathrm{C} \& \mathrm{~F}-1$ coop and even better when using C\&F-MultiCoop. The difference between DC-ARQ and C\&F-MultiCoop is reduced for high download nodes density (scen.-A) because there are few packets cooperated. We could also notice that for sparser node's density scenarios (scen.-D) C\&F-1 coop and C\&F-MultiCoop have nearly the same behavior because the probability of several nodes being in AP coverage diminishes. In sparsest scenarios it is when C\&F mechanisms take advantage of APs not being used. Then, it must be noticed that while the gain obtained by DC-ARQ cooperation is more relevant at denser scenarios, C\&F cooperation is more relevant at sparser scenarios, so using both of them we gain synergy because the framework is ready to increase the performance in both scenarios.

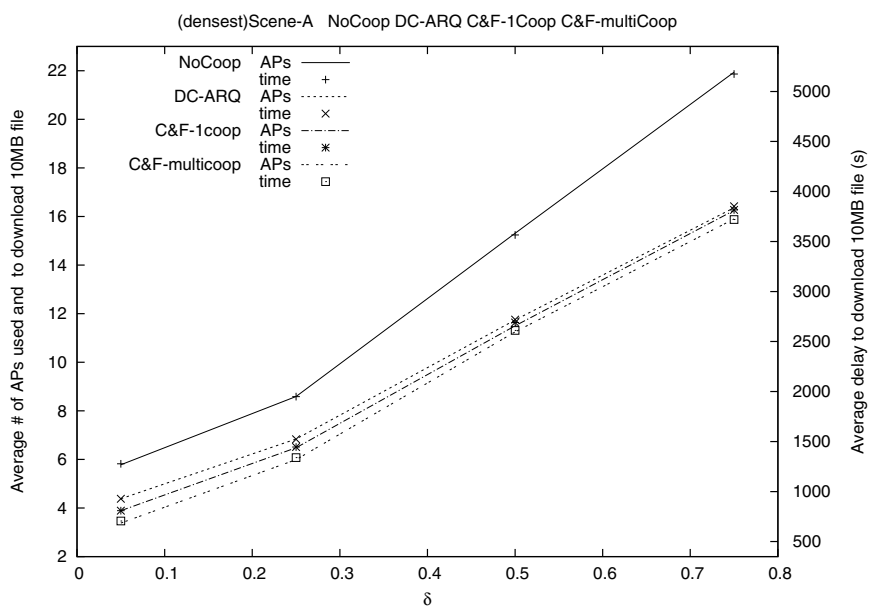

Figure 7. Average \# of APs used with each mechanism in Scene-A

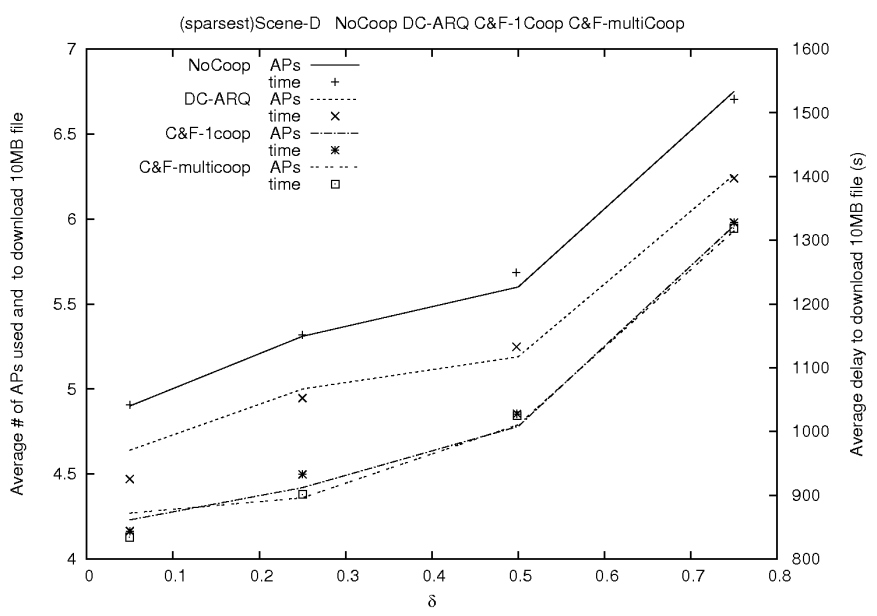

Figure 8. Average \# of APs used with each mechanism in Scene-D

\section{CONCLUSIONS}

In this paper it has been proposed a vehicular framework that opportunistically allows downloading packets when vehicles cross AP. The framework works as a Delay Tolerant Network and benefits two cooperative mechanisms: (i) a DC-
ARQ to recover packet losses due to the harsh physical conditions and (ii) a carry and forward mechanism to improve throughput and total transfer delay. We have shown how the combination of both mechanisms improves these performance parameters depending on vehicle density and number of vehicles downloading packets. Dense vehicle scenarios make better use of DC-ARQ while sparse vehicle scenarios make better use of carry and forward mechanisms. We expect the same kind of behavior in more complex road scenarios, i.e. with bifurcations or urban scenarios, since the results do not depend on the number of neighboring AP. However, in these scenarios a better coordination among neighbor AP will be needed, for instance in terms of vehicle travel prediction.

\section{REFERENCES}

[1] J. Ott, D. Kutscher, "Drive-thru Internet: IEEE $802.11 \mathrm{~b}$ for 'Automobile' users", IEEE INFOCOM 2004.

[2] J. Morillo, J. Garcia-Vidal, "A Low Coordination Overhead C-ARQ Protocol with Frame Combining", 18th Annual IEEE International Symposium on Personal, Indoor and Mobile Radio Communications (IEEE PIMRC 2007).

[3] J. Morillo-Pozo, J. García-Vidal, A. Pérez-Neira, "Collaborative ARQ in Wireless Energy-Constrained Networks", ACM-SIGMOBILE DIAL-MPOMC International Workshop on Foundation of Mobile Computing, September 2, 2005, Cologne, Germany.

[4] J. Morillo-Pozo, J. M. Barcelo-Ordinas, O. Trullos-Cruces, J. GarciaVidal, "A Cooperative ARQ for Delay Tolerant Vehicular Networks", 3rd IEEE International Workshop on Delay/Disruption-Tolerant Mobile Networks (DTMN'08), in conjunction with ICDCS 2008, Beijing, China, June 20, 2008

[5] Bin Zhao, Matthew C. Valenti, "Practical Relay Networks: A Generalization of Hybrid-ARQ", IEEE Journal on Selected Areas in Communications, vol. 23, no. 1, Jan. 2005.

[6] A. Miu, H. Balakrishnan, C. E. Koksal, "Improving Loss Resilience with Multi-Radio Diversity in Wireless Networks", ACM Mobicom 2005, September 2005.

[7] M. Dianati, X. Ling, K. Naik, X. Shen, "A Node Cooperative ARQ Scheme for Wireless Ad-hoc Networks", IEEE Wireless Communications and Networking Conference, 2005.

[8] J. Burguess, B. Gallagher, D. Jensen and B.N. Levine, "MaxProp: Routing for Vehicle-based Disruption Tolerant Networks", 25th Conference on Computer Communications (IEEE INFOCOM), Barcelona, Spain, April 2006

[9] A. Balasubramanian and B. N. Levine and A. Venkataramani, "DTN Routing as a Resource Allocation Problem", ACM SIGCOM'07, Kyoto, Japan, August 2007.

[10] J. Zhao and G. Cao, "VADD: Vehicle-Assisted Data Delivery in Vehicular Ad Hoc Networks", 25th Conference on Computer Communications (IEEE INFOCOM), Barcelona, Spain, April 2006.

[11] A. Vahdat and D. Becker, "Epidemic Routing for Partially Connected Ad Hoc Networks" Technical Report CS-200006, Duke University, April 2000.

[12] A. Nandan, S. Das, G. Pau, M. Gerla, M.Y. Sanadidi, "Co-operative downloading in vehicular ad-hoc wireless networks", WONS, Jan 2005

[13] K. Fall, "A Delay-Tolerant Network Architecture for Challenge Internets", in ACM SIGCOMM 2003, Aug. 2003.

[14] A. Lindgren, A. Doria and O. Schelen, "Probabilistic routing in intermittently connected networks", The First International Workshop on Service Assurance with Partial and Intermittent Resources (SAPIR 2004) and also as a Poster in MobiHoc'03, Aug 2004.

[15] Network Simulator ns-2. http://www.isi.edu/nsnam/ns

[16] M. Torrent-Moreno, "Inter-Vehicle Communications: Achieving Safety in a Distributed Wireless Environment", Dissertation, Universitätsverlag Karlsruhe, 2007. 